\title{
Recent advances in the $\mu-e$ scattering amplitude at NNLO in QED
}

\author{
William J. Torres Bobadilla*† \\ Instituto de Física Corpuscular, Universitat de València - Consejo Superior de Investigaciones \\ Científicas, \\ Parc Científic, E-46980 Paterna, Valencia, Spain \\ E-mail: william.torres@ific.uv.es
}

In this talk, we review the recent developments of the next-to-next-to leading order contribution to the $\mu e$-elastic scattering. We focus our discussion on the interference between the two-loop and the Born amplitude. In particular, we discuss the analytic reduction of the two-loop amplitude by means of the integrand reduction methods and the application of the integration-by-parts identities. On top of it, we show preliminary results for the ultraviolet renormalisation of the latter.

European Physical Society Conference on High Energy Physics - EPS-HEP2019 -

10-17 July, 2019

Ghent, Belgium

\footnotetext{
* Speaker.

${ }^{\dagger}$ Talk based on a collaboration with S. Di Vita, S. Laporta, P. Mastrolia, M. Passera, T. Peraro, A. Primo, J. Ronca and U. Schubert.
} 


\section{Introduction}

In this talk we review the recent developments of the evaluation of the two-loop virtual correction to the electron-muon scattering, $\mu e \rightarrow \mu e$, at Next-to-Next-to-Leading order in QED. These radiative corrections are relevant for the analysis of the MUonE experiment, recently proposed at CERN. MUonE aims at the high precision determination of the QED running coupling constant in the space-like region from the measurement of the differential cross section of the elastic scattering of high-energy muons on atomic electrons. We focus our discussion on the implementation of the adaptive integrand decomposition algorithm (AIDA) $[1,2]$ and the interplay with available tools for the reduction and computation of multi-loop integrals. Furthermore, we comment on the progress made towards the renormalisation of the amplitude.

This contribution is organised as follows. In Sec. 2 we briefly remark the main features of the adaptive integrand decomposition algorithm (AIDA). Then, in Sec. 3, we recall the organisation to reduce the two-loop amplitude. In Sec. 4, we discuss and summarise all the work done for the unrenormalised amplitude. The preliminary results towards the UV renormalisation is displayed in Sec. 5 . We finally draw our summary of the talk.

\section{Adaptive Integrand Decomposition in a nutshell}

In this section, we explain the main features of the Adaptive Integrand Decomposition Algorithm (AIDA) [1,2]. We remark that this method decomposes the space-time dimension, $d=4-2 \varepsilon$, into parallel (or longitudinal) and orthogonal (or transverse) dimensions, $d=d_{\|}+d_{\perp}$. Parallel and orthogonal directions show particular properties for topologies with less than five external legs.

In the structure of the Feynman integrals,

$$
\mathscr{I}_{i_{1} \cdots i_{n}}^{(\ell)}[\mathscr{N}]=\int\left(\prod_{i=1}^{\ell} \frac{d^{d} \bar{l}_{i}}{\pi^{d / 2}}\right) \frac{\mathscr{N}_{i_{1} \cdots i_{n}}\left(\bar{l}_{i}\right)}{\prod_{j} D_{j}\left(\bar{l}_{j}\right)}
$$

loop momenta become

$$
\bar{l}_{i}^{\alpha}=l_{\| i}^{\alpha}+\lambda_{i}^{\alpha},
$$

with

$$
\bar{l}_{\| i}^{\alpha}=\sum_{j=1}^{d_{\|}} x_{j i} e_{j}^{\alpha}, \quad \lambda_{i}^{\alpha}=\sum_{j=d_{\|}+1}^{4} x_{j i} e_{j}^{\alpha}+\mu_{i}^{\alpha}, \quad \lambda_{i j}=\sum_{l=d_{\|}+1}^{4} x_{l i} x_{l j}+\mu_{i j} .
$$

In Eq. (2.2), $l_{\| i}$ is a vector of the $d_{\|}$-dimensional space spanned by the external momenta, and $\lambda_{i}$ belongs the $d_{\perp}$-dimensional orthogonal subspace. In this parametrisation, all denominators become independent of the transverse components of the loop momenta.

Let us indicate with $\mathbf{z}$ the full set of $\ell(\ell+9) / 2$ variables

$$
\mathbf{z}=\left\{\mathbf{x}_{\| i}, \mathbf{x}_{\perp i}, \lambda_{i j}\right\}, \quad i, j=1, \ldots \ell,
$$

where $\mathbf{x}_{\| i}\left(\mathbf{x}_{\perp i}\right)$ are the components of the loop momenta parallel (orthogonal) to the external kinematics, the denominators are reduced to polynomials in the subset of variables

$$
\tau=\left\{\mathbf{x}_{\|}, \lambda_{i j}\right\}, \quad \tau \subset \mathbf{z},
$$


so that the general $r$-point integrand has the form

$$
\mathscr{I}_{i_{1} \ldots i_{r}}\left(\tau, \mathbf{x}_{\perp}\right) \equiv \frac{\mathscr{N}_{i_{1} \ldots i_{r}}\left(\tau, \mathbf{x}_{\perp}\right)}{D_{i_{1}}(\tau) \cdots D_{i_{r}}(\tau)} .
$$

Since numerator and denominators depend on different variables, the adaptive integrand decomposition suggests the following algorithm:

1. Divide: we divide the numerator $\mathscr{N}_{i_{1} \ldots i_{r}}\left(\tau, \mathbf{x}_{\perp}\right)$ modulo the Gröbner basis $\mathscr{G}_{i_{1} \ldots i_{r}}(\tau)$ of the ideal $\mathscr{J}_{i_{1} \cdots i_{r}}(\tau)$ generated by the set of denominators. The polynomial division is performed be adopting the lexicographic ordering $\lambda_{i j} \ll \mathbf{x}_{\|}$,

$$
\mathscr{N}_{i_{1} \ldots i_{r}}\left(\tau, \mathbf{x}_{\perp}\right)=\sum_{k=1}^{r} \mathscr{N}_{i_{1} \ldots i_{k-1} i_{k+1} \ldots i_{r}}\left(\tau, \mathbf{x}_{\perp}\right) D_{i_{k}}(\tau)+\Delta_{i_{1} \ldots i_{r}}\left(\mathbf{x}_{\|}, \mathbf{x}_{\perp}\right)
$$

The Gröbner basis does not need to be explicitly computed, since, with the choice of variables and the ordering described here, the division is equivalent to applying the set of linear relations described above.

2. Integrate: Since denominators do not depend on transverse variables, $\mathbf{x}_{\perp}$, we can integrate the residue $\Delta_{i_{1} \ldots i_{r}}$ over transverse directions. This integration is carried out by expressing $\Delta_{i_{1} \ldots i_{r}}$ in terms of Gegenbauer polynomials, i.e.,

$$
\Delta_{i_{1} \ldots i_{r}}^{\mathrm{int}}(\tau)=\int d^{\left(4-d_{\|}\right) \ell} \Theta_{\perp} \Delta_{i_{1} \ldots i_{r}}\left(\tau, \Theta_{\perp}\right)
$$

Where $\Delta_{i_{1} \ldots i_{r}}^{\mathrm{int}}$ is a polynomial in $\tau$ whose coefficients depend on the space-time dimension $d$.

3. Divide: the structure of the integrated residue suggests a second division. This can be seen from the dependence $\Delta_{i_{1} \ldots i_{r}}^{\mathrm{int}}$ has on the variables $\tau$. In fact, after applying the division, similarly as in the first step of this algorithm, we get

$$
\Delta_{i_{1} \ldots i_{r}}^{\mathrm{int}}(\tau)=\sum_{k=1}^{r} \mathscr{N}_{i_{1} \ldots i_{k-1} i_{k+1} \ldots i_{r}}^{\mathrm{int}}(\tau) D_{i_{k}}(\tau)+\Delta_{i_{1} \ldots i_{r}}^{\prime}\left(\mathbf{x}_{\|}\right)
$$

where the new residue $\Delta_{i_{1} \ldots i_{r}}^{\prime}\left(\mathbf{x}_{\|}\right)$can only depend on $\mathbf{x}_{\|}$.

\section{3. $\mu e$ elastic scattering}

Motivated by the new experiment MUonE proposed at CERN [3] that provides a new and independent determination of the leading hadronic contribution to the muon $g-2$ [3, 4], we consider as an application of AIDA, the two-loop reductions of the $\mu e$ elastic scattering,

$$
e^{-}\left(-p_{1}\right) \mu^{-}\left(-p_{4}\right) \rightarrow e^{-}\left(p_{2}\right) \mu^{-}\left(p_{3}\right)
$$

The electron is treated as massless, $m_{e}^{2}=0$, while we retain full dependence on the muon mass, $m_{\mu}^{2} \neq 0$. We focus on the spin summed/averaged squared matrix elements,

$$
M^{(1)}=2 \operatorname{Re}\left\langle A_{e \mu}^{(0)} \mid A_{e \mu}^{(1)}\right\rangle, \quad M^{(2)}=2 \operatorname{Re}\left\langle A_{e \mu}^{(0)} \mid A_{e \mu}^{(2)}\right\rangle,
$$




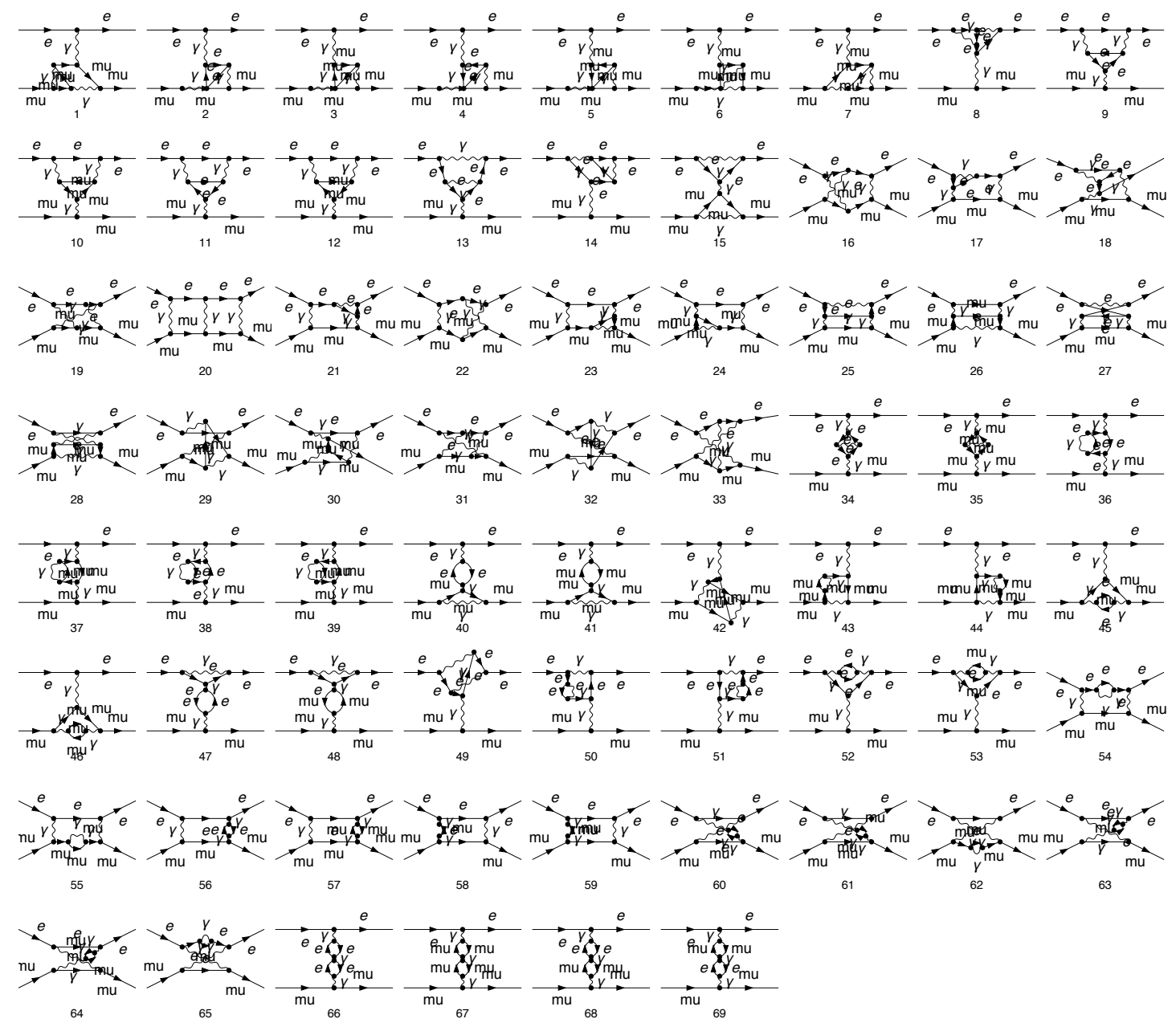

Figure 1: Feynman diagrams contributing to the two-loop $\mu e$-scattering amplitude.

to obtain the results of the corresponding two-loop amplitude.

Hence, we define the kinematical variables this amplitude depends on to be

$$
s=\left(p_{1}+p_{2}\right)^{2}, \quad t=\left(p_{2}+p_{3}\right)^{2}, \quad u=\left(p_{1}+p_{3}\right)^{2}=-s-t+2 m_{\mu}^{2} .
$$

For the calculation of the two-loop amplitude of the $\mu e$ elastic scattering, we have to evaluate the Feynman diagrams of Fig 1. Since we are interested in the reduction of the integrand by means of AIDA, we apply the algorithm divide-integrate-divide as described in the previous sections. These steps can be summarised as follows.

1. Identify parent topologies and group diagrams:

$$
\begin{aligned}
& \{\{1,46\},\{3,5\},\{6,35,41,67\},\{7\},\{16\},\{17,8,36,38,50,52,54,58\},\{18,60,65\},\{19\}, \\
& \{20\},\{21,14,51,56\},\{22\},\{23,57\},\{24,55,59\},\{25,2,4,9,11,13,34,47,66\}, \\
& \{26,10,12,15,37,39,40,43,44,48,68,69\},\{27\},\{28\},\{29,61,62\},\{30,64\},\{31\}, \\
& \{32,63\},\{33\},\{42\},\{45\},\{49\},\{53\}\} .
\end{aligned}
$$


2. Generate cuts: let us focus on the sixth group, whose parent topology

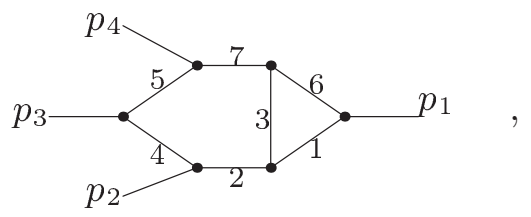

gives contribution to the following cuts

$$
\begin{aligned}
& \{\{\{1,6\},\{2,4,5,7\},\{3\}\},\{\{6\},\{2,4,5,7\},\{3\}\},\{\{1,6\},\{4,5,7\},\{3\}\},\{\{1,6\},\{2,4,5,7\}\}, \\
& \{\{1,6\},\{2,5,7\},\{3\}\},\{\{1,6\},\{2,4,7\},\{3\}\},\{\{1\},\{2,4,5,7\},\{3\}\},\{\{1,6\},\{2,4,5\},\{3\}\}, \\
& \{\{6\},\{4,5,7\},\{3\}\},\{\{6\},\{2,4,5,7\}\},\{\{6\},\{2,5,7\},\{3\}\},\{\{6\},\{2,4,7\},\{3\}\},\{\{2,4,5,7\}, \\
& \{3\}\},\{\{6\},\{2,4,5\},\{3\}\},\{\{1,6\},\{4,5,7\}\}, \ldots,\{\{1\},\{3\}\},\{\{1\},\{2\}\}\} .
\end{aligned}
$$

4. Organise cuts in jobs: in order to consider the full contribution of all possible diagrams, there is a subtlety w.r.t. the one-loop case, which relies on the treatment of the diagrams that contain squared propagators, e.g. diagrams 36-58 in Fig. 1. For instance, the contribution to the cut $\{\{1,6\},\{2,4,5\},\{3\}\}$ with linear propagators gets contributions from

$$
\left\{\mathbf{N}_{\{\{1,6\},\{2,5,7\},\{3\}\}}^{\{\{1,6\},\{2,4,5,\{3\}\}}, \mathbf{N}_{\{\{1,6\},\{2,5,7\},\{3\}\}}^{\{1,6\},\{\dot{2}, 5,7\},\{3\}\}}, \mathbf{N}_{\{\{1,6\},\{2,5,5,7\},\{3\}\}}^{\{1,6\},\{2,5,7\},\{3\}\}},\right.
$$

where superscripts with dots represent squared propagators.

\section{Discussion on the integrand reduction of $e \mu$}

Let us briefly discuss a few features of the two-loop calculation we are performing w.r.t. the one-loop case. At the one-loop level in AIDA's framework, as described in [5-7], it is possible to reduce the amplitude or any one-loop object to a linear combination of the standard master integrals, i.e. boxes, triangles, bubbles and tadpoles, where all of them are known either analytically or numerically. At the two-loop level, on the contrary, the inclusion of irreducible scalar products, not present at one-loop, does not allow to end up with scalar integrals whose numerator is 1 . Then, relation at integral level, given by the symmetry of the latter, are used in order to reduce the number of integrals to evaluate.

The reduction of integrals is known as integration-by-parts (IBP) identities [8-10]. Specifically for the calculation we are performing, we manage to reduce the number of integrals to evaluate from $\mathscr{O}(10000) \rightarrow \mathscr{O}(100)$. Due to the flexibility of AIDA, it is straightforward to interface the output the latter generates to later pass to any software that generates IBPs. In fact, These two kind of reductions, integrand and IBP, are done independently. One just needs information of the external kinematics and the structure of the topology to reduce. For our purposes we use REDUZE [11] and KIRA [12].

Furthermore, for processes at multi-loop level, one needs to deal with the kind of topology, planar or non-planar. The latter was not present at one-loop level. Despite the proliferation of terms appearing at two-loop level, there has been an enormous effort for computing the integrals needed to generate the, to begin with, unrenormalised amplitude. In fact, their calculation has been split 
into two contributions. i) The calculation of the integrals originated from the planar topologies [13] and ii) the ones for non-planar topologies [14].

From the above discussion, we claim that the evaluation of the unrenormalised amplitude is at hand. What is next? The UV renormalisation of the latter. In order to do it, we renormalise as follows. We renormalise in a scheme where fermion masses are pole masses and the gauge coupling is in the $\overline{\mathrm{MS}}$ scheme. To this end, we renormalise fields and couplings in $\overline{\mathrm{MS}}$ except for the fermion masses, for which we would use the pole mass prescription, and to separately include finite Lehmann, Symanzik and Zimmermann (LSZ) factors for the external legs [15].

\section{Preliminary results on the UV renormalisation}

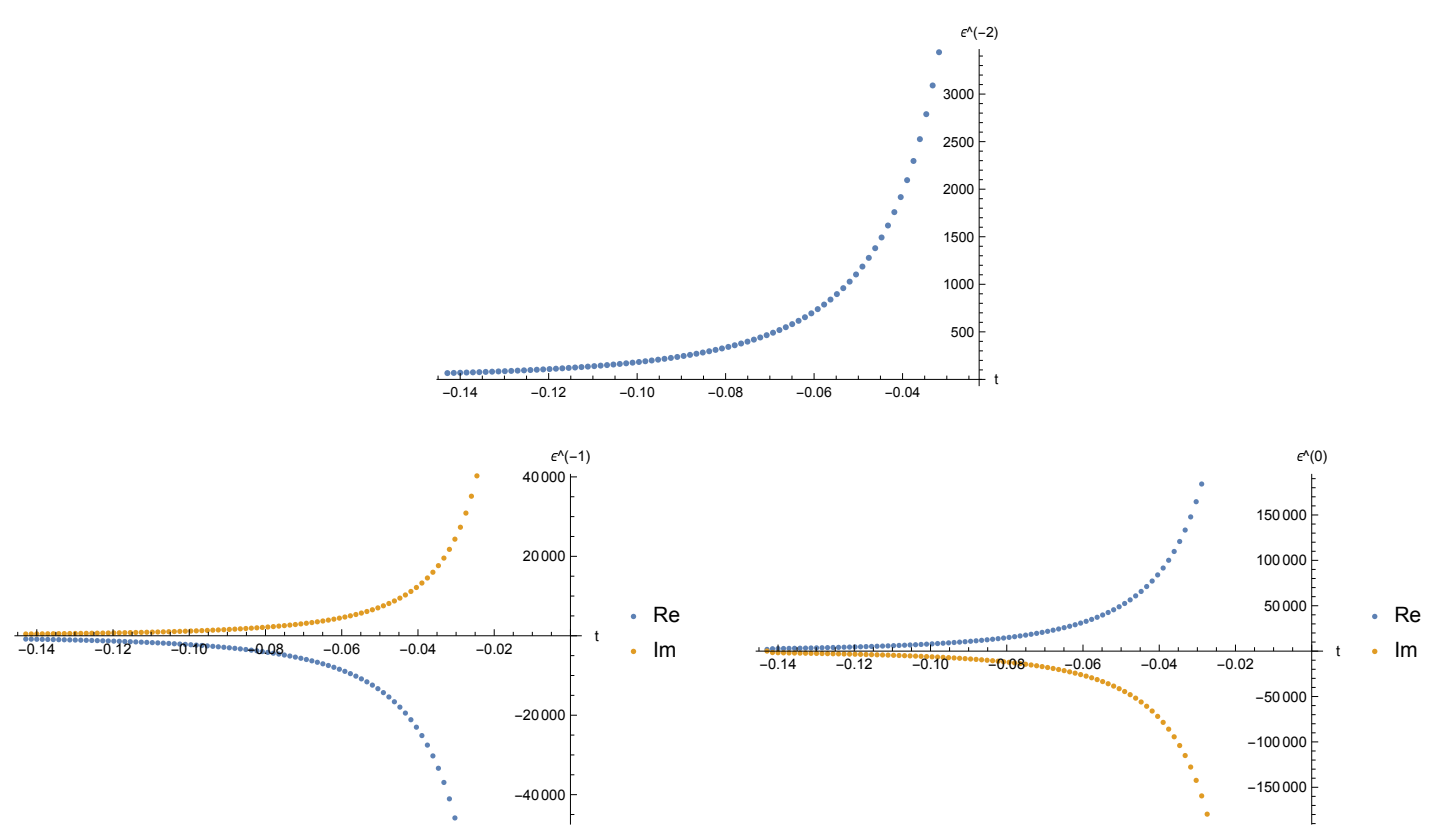

Figure 2: Results for the UV renormalised diagram 58.

In this section, we display the first results towards the renormalisation of the scattering amplitude $e \mu \rightarrow e \mu$ at two-loop level. The very first exercise we do is to renormalise the diagram 58 of Fig. 1, in which we use this kinematics:

$$
\begin{aligned}
\sqrt{s}=0.40554 \mathrm{GeV}, & m & =105.6583745 \mathrm{MeV}, \\
\text { scan over } t_{\min } \leq t<0, & t_{\min } & =0.142893 \mathrm{GeV}^{2} .
\end{aligned}
$$

With the on-shell renormalisation procedure we cancel the leading pole $1 / \varepsilon^{3}$, leaving the next-toleading poles, whose behaviour is shown in Fig. 2.

\section{Conclusions}

We discussed the calculation of the two-loop scattering amplitude $e \mu \rightarrow e \mu$, where we remarked the main features that were done at integrand and integral level. On top of it, we showed preliminary results toward the complete calculation of the renormalised amplitude. 


\section{Acknowledgments}

This work is supported by the Spanish Government (Agencia Estatal de Investigación) and ERDF funds from European Commission (Grants No. FPA2017-84445-P and SEV-2014-0398), Generalitat Valenciana (Grant No. PROMETEO/2017/053), Consejo Superior de Investigaciones Científicas (Grant No. PIE-201750E021), the COST Action CA16201 PARTICLEFACE and the "Juan de la Cierva Formación” program (FJCI-2017-32128).

The Feynman diagrams depicted in this paper were generated using FEYNARTs [16].

\section{References}

[1] P. Mastrolia, T. Peraro, and A. Primo JHEP 08 (2016) 164, [1 605 . 0315 7].

[2] P. Mastrolia, T. Peraro, A. Primo, and W. J. Torres Bobadilla PoS LL2016 (2016) 007, [1607.05156].

[3] G. Abbiendi et al. Eur. Phys. J. C77 (2017), no. 3 139, [1609. 08987 ].

[4] C. M. Carloni Calame, M. Passera, L. Trentadue, and G. Venanzoni Phys. Lett. B746 (2015) 325-329, [1504.02228].

[5] W. J. Torres Bobadilla PoS LL2018 (2018) 036.

[6] P. Mastrolia, M. Passera, A. Primo, U. Schubert, and W. J. Torres Bobadilla EPJ Web Conf. 179 (2018) 01014.

[7] W. J. Torres Bobadilla PoS RADCOR2017 (2018) 082, [1801. 03010 ].

[8] F. V. Tkachov Phys. Lett. B100 (1981) 65-68.

[9] K. G. Chetyrkin and F. V. Tkachov Nucl. Phys. B192 (1981) 159-204.

[10] S. Laporta Int. J. Mod. Phys. A15 (2000) 5087-5159, [hep-ph / 0102033$].$

[11] A. von Manteuffel and C. Studerus 1201.4330.

[12] P. Maierhfer, J. Usovitsch, and P. Uwer Comput. Phys. Commun. 230 (2018) 99-112, [1705.05610].

[13] P. Mastrolia, M. Passera, A. Primo, and U. Schubert JHEP 11 (2017) 198, [1709. 07435 ].

[14] S. Di Vita, S. Laporta, P. Mastrolia, A. Primo, and U. Schubert JHEP 09 (2018) 016, [1806. 08241 ].

[15] H. Lehmann, K. Symanzik, and W. Zimmermann Il Nuovo Cimento (1955-1965) 1 (Jan, 1955) $205-225$.

[16] T. Hahn Comput. Phys. Commun. 140 (2001) 418-431, [hep-ph / 0012260$].$ 